Cahiers d'études africaines

163-164|2001

Langues déliées

\title{
À la découverte des amhariques
}

Langues et histoires éthiopiennes en regard

\section{Eloi Ficquet}

\section{OpenEdition}

\section{Journals}

Édition électronique

URL : http://journals.openedition.org/etudesafricaines/106

DOI : 10.4000/etudesafricaines.106

ISSN : $1777-5353$

Éditeur

Éditions de l'EHESS

Édition imprimée

Date de publication : 1 janvier 2001

Pagination : 497-516

ISBN : 978-2-7132-1394-6

ISSN : 0008-0055

\section{Référence électronique}

Eloi Ficquet, «À la découverte des amhariques », Cahiers d'études africaines [En ligne], 163-164 | 2001, mis en ligne le 31 mai 2005, consulté le 05 février 2021. URL : http://journals.openedition.org/ etudesafricaines/106 ; DOI : https://doi.org/10.4000/etudesafricaines.106 


\title{
Éloi Ficquet
}

\section{À la découverte des amhariques}

\author{
Langues et histoires éthiopiennes en regard
}

Mettre en évidence l'hétérogénéité des pratiques linguistiques des locuteurs d'amharique et la diversité des espaces sociaux dans lesquels ils opèrent des contacts avec d'autres langues, implique de déstabiliser, sans chercher à l'abattre, l'un des piliers de la construction nationale éthiopienne. Que ce soit dans la version centralisatrice de cet État, ou dans la version fédérale contemporaine, l'amharique a été au $\mathrm{Xx}^{\mathrm{e}}$ siècle la «langue des langues » en Éthiopie.

Dans cette démarche, il faut aussi tenir compte du poids des traditions savantes occidentales, qui ont créé dans les études éthiopisantes une forte démarcation entre les domaines d'étude des orientalistes et des africanistes. Les premiers se sont en effet intéressés aux sociétés à dominante chrétienne des hautes terres du Nord pour leur histoire politique et religieuse, conservée par de rares manuscrits, et pour leurs langues apparentées au groupe dit sémitique (dont l'amharique). Ils laissaient aux seconds le champ libre pour étudier les sociétés périphériques à tradition orale, sur lesquelles ont longtemps pesé de forts préjugés d'inculture et de barbarie. L'existence d'un tel clivage a sans doute limité, de part et d'autre, la profondeur et la pertinence des enquêtes et des analyses, cependant la prise de conscience de cette dualité a aussi suscité des recherches transversales souvent novatrices et de plus en plus nombreuses.

Cet article propose de pénétrer dans le «bastion» orientaliste des travaux sur l'amharique, afin de rendre compte d'abord des représentations qu'ils ont produites ou répercutées, pour tenter ensuite d'élargir le champ d'observation de cette langue et des gens qui la parlent. Notre contribution n'est pas fondée sur une étude systématique des activités langagières en amharique, mais sur des enquêtes anthropologiques parmi des Éthiopiens qui ont un usage exclusif, courant ou occasionnel de cette langue. Nous n'établirons ici que peu de faits linguistiques, mais en nous écartant des normes minutieuses de description grammaticale ou lexicale, nous inciterons à y revenir avec des hypothèses renouvelées.

Le présupposé qu'une langue ne peut être étudiée que comme un système clos ayant sa cohérence interne, a été contrebalancé par la prise en 
compte de la pluralité des interactions entre les langues et, depuis plus récemment, par l'observation des rapports du sujet communiquant aux réalités plurilingues dans lesquelles il évolue. Cependant, l'unicité d'une langue n'est pas une pure illusion produite par la description scientifique. Quand bien même cette unicité serait construite par d'autres représentations, discours ou pratiques, il faut pouvoir en rendre compte, avec les mêmes outils d'analyse que ceux mis en œuvre dans l'étude de la diversité des agencements linguistiques. Une langue est en contact avec elle-même autant qu'avec d'autres. L'amharique présente ainsi une forte apparence d'uniformité, prise comme un fait acquis par ses descripteurs, mais qui a été aussi peu mise en question que les problèmes d'hétérogénéité à différents niveaux, dialectal, interlinguistique et historique.

En commençant par examiner ces dernières questions, nous montrerons que les conceptions génétiques et diffusionnistes de l'amharique sont liées à des représentations identitaires et idéologiques qui ont usé d'arguments linguistiques et les ont nourris en retour. Il est néanmoins possible d'échapper à ce cercle en prenant des tangentes en divers points. Nous avons choisi de présenter ici quelques considérations sur une langue parente de l'amharique parlée par des communautés musulmanes établies sur le rebord des hauts plateaux centraux d'Éthiopie. Cette perspective nous permettra de mettre au jour une zone d'interface culturelle et linguistique très active, dont l'ancienneté nous amènera à reconsidérer les liens admis entre l'extension de la langue amharique et l'histoire politique éthiopienne.

\section{Un déni d'hétérogénéité}

Il est peu de langues qui semblent présenter aussi peu de variations dialectales que l'amharique. Un seul article, collectif, est paru sur la question en 1973 (Habte Mariam Marcos 1973), repris trois ans plus tard par d'autres auteurs dans un ouvrage consacré aux langues éthiopiennes (Bender et al. 1976 : 90-98). Après des enquêtes de très courte durée, menées en 1969, quatre équipes de linguistes de l'Université d'Addis Abeba avaient établi des comparaisons entre des dialectes régionaux (gojjam, wällo, mänz et gondär) et celui, mal identifié, de la capitale éthiopienne. Les trois comptes rendus finalement publiés suggéraient une certaine diversité de formes à différents niveaux d'analyse, mais restaient sur une approche assez superficielle, en proposant d'entreprendre des recherches plus extensives, qui sont restées sans suite. Le désintérêt, voire le déni des linguistes de l'amharique à l'encontre de la question dialectale, correspond à une représentation largement diffusée d'une uniformité de cette langue. Nous reviendrons plus bas sur les implications politiques et historiques de cette question.

Les emprunts lexicaux de l'amharique, identifiés dans les dictionnaires ou dans les néologismes, ont suscité quelques notes lexicographiques érudites, fournissant des analyses plus ou moins profondes des dynamiques 
interlinguistiques révélées par ces items (Tubiana 1984, 1985, 1986 ; Leslau 1990 ; Bender 1972). En outre, quelques contributions ont approché la question des contacts entre langues en Éthiopie d'un point de vue sociolinguistique (Drewes 1970 ; Ferguson 1971 ; Amsalu Aklilu 1991), mais en proposant de très maigres observations et en effleurant les problèmes sociologiques et historiques qui apparaissaient. À notre connaissance, aucune enquête n'a été conduite ni même projetée sur des mixtes linguistiques possibles impliquant l'amharique, même sur des terrains favorables à cette entreprise. Malgré l'abondance des travaux sur les Falasha et leur intégration à la société israélienne, il ne semble pas y avoir eu d'études sur leur amharique au contact de l'hébreu talmudique et moderne. De même pour l'amharique parlé par d'autres diasporas éthiopiennes en contact avec des langues et des cultures occidentales. L'amharique pratiqué à Addis Abeba, depuis plus d'un siècle d'existence de cette capitale au carrefour des identités éthiopiennes, n'a pas non plus encore fait l'objet de descriptions.

\section{Un creuset linguistique originel}

C'est surtout en des termes génétiques que le problème de l'hétérogénéité linguistique a été posé sur l'amharique, et sur les langues éthiopiennes en général, par la reconstruction de relations entre des proto-langues dont la synthèse aurait produit les langues actuellement observables. L'amharique et les autres langues éthiopiennes apparentées au groupe sémitique dériveraient de langues homologues sudarabiques ou arabiques, transformées au contact de langues classifiées comme couchitiques, qu'auraient parlées les peuples indigènes, pré-sémitiques, de la Corne de l'Afrique. La reconstruction de ces « relations de parenté » entre langues éthiopiennes a fait l'objet de recherches et de débats entre philologues et linguistes depuis près d'un siècle et demi, contribuant particulièrement au comparatisme chamitosémitique.

Le guèze (ge'ez) est la plus ancienne des langues «éthio-sémitiques » connues. Parlée par les sujets du royaume antique d'Aksum (formé au $\mathrm{I}^{\mathrm{er}}$ siècle, converti au christianisme au $\mathrm{IV}^{\mathrm{e}}$ ), elle semble s'être éteinte avec cet État vers le $\mathrm{x}^{\mathrm{e}}$ siècle. Elle fut cependant conservée comme langue littéraire du royaume dit salomonide ${ }^{1}$ jusqu'au XIX ${ }^{\mathrm{e}}$ siècle, et comme langue liturgique du clergé monophysite ${ }^{2}$ éthiopien jusqu'à aujourd'hui.

La connaissance du guèze est à la base du comparatisme des langues sémitiques éthiopiennes, dans deux directions. En amont, ont été recherchées les filiations à d'autres langues sémitiques anciennes, en particulier aux

1. C'est-à-dire dirigé par une dynastie qui se prétend être de la descendance de Salomon.

2. Qui reconnaît l'unité de la personne du Christ dans sa nature divine, qui a absorbé la nature humaine. C'est ainsi une apparence du Christ qui a été crucifiée selon ce dogme. 
langues sudarabiques. Les traces épigraphiques du guèze permettent en effet de remonter à des états anciens de cette langue et d'affiner les comparaisons avec les langues écrites des royaumes antiques d'Arabie du Sud. En aval, les langues éthiopiennes contemporaines, identifiées comme sémitiques, ont d'abord été comparées au guèze, puis entre elles. Les travaux entrepris dans cette dernière direction ont été inaugurés par le grand savant Hiob Ludolf, auteur de la première grammaire de l'amharique (1698). Parmi ses nombreux successeurs, auteurs d'une masse considérable de travaux d'érudition, Franz Praetorius a établi le premier l'histoire et l'étymologie de l'amharique par une analyse comparative (1879). Marcel Cohen a élargi ce champ d'investigation à d'autres langues et considérablement amplifié cette méthode, notamment dans ses Études d'éthiopien méridional (1931), qui ont préludé à la parution de son monumental Traité de langue amharique (1936), dernière description complète de cette langue en Europe ${ }^{3}$. Dans cet ouvrage, il résume les hypothèses sur la nature historique de l'amharique : «Par son caractère linguistique, il semble être une forme passablement évoluée d'un parler proche mais distinct du guèze. Son degré d'évolution lui donne une situation analogue à celle par exemple du français parmi les langues romanes. On peut expliquer la plupart de ses formes, mais non toutes, en le comparant au guèze ; sa position serait comparable moins à celle du français par rapport au latin qu'à celle de l'allemand par rapport au gotique. Il a subi fortement l'influence d'un substrat couchitique » (Cohen 1936: 13).

Cette notion de substrat couchitique est en partie fondée sur des indices fournis par l'analyse comparée avec des lexiques de langues éthiopiennes non sémitiques. Les comparaisons les plus fertiles ont porté sur un ensemble culturel et linguistique très fragmenté, dit Agäw, formé d'isolats plus ou moins peuplés, pris dans la masse éthio-sémitique ${ }^{4}$. Leur observation a révélé des échanges lexicaux anciens entre ce groupe de langues, dont on suppose qu'elles furent beaucoup plus étendues, et les langues sémitiques qui les auraient supplantées. Cette théorie a été confirmée par des études plus récentes s'appuyant sur des matériaux descriptifs plus précis (Bender 1971 ; Appleyard 1978, 1979). Néanmoins le processus d'extinction de ces langues, les étapes de leur recouvrement par les langues aujourd'hui majoritaires restent encore mal connues. L'extension d'un État puissamment organisé, fondé sur une haute culture lettrée, connecté par la religion à des réseaux de pensée et de pouvoir universels, absorbant et assimilant dans sa masse toujours plus grande, toujours plus forte, des sociétés gravitant autour de lui est l'hypothèse la plus communément admise, mais réductrice. Cette vue dialectique de l'histoire réduit chacun de ces ensembles linguistiques

3. Wolf Leslau, disciple de Cohen, professeur émérite à l'Université of California at Los Angeles, a récemment produit une imposante grammaire de l'amharique, dont la composition est très proche de celle du Traité.

4. Les principaux groupes étant les Awngi au Sud, les Khamir et les Khamta à l'Est, les Kemant à l'Ouest, les Bilin au Nord, voir Tubiana (1959). 
à deux rôles antagonistes, les uns participant d'un degré de civilisation supérieur aux autres, sauvages, désorganisés et dominés : «Les peuples couchitiques sont installés dans la Corne de l'Afrique depuis des millénaires. Ils forment l'élément ethnique [i.e. racial] de base de la population d'Éthiopie. [...] Les apports continus d'éléments sémitiques provenant d'Arabie du SudOuest, avant et après l'ère chrétienne, ont recréé une union des Hamites et des Sémites, sur la scène des hauts plateaux abyssins. Au terme de cette nouvelle fusion, les Hamites sont apparus comme le facteur ethnique prédominant et les Sémites comme le principal fonds d'éléments culturels et linguistiques » (Ullendorf $1973: 43$ ).

Ce type de représentation, que l'on retrouve dans d'autres contextes historiques, s'est élaboré dans le cas éthiopien à partir de prénotions, certaines fort anciennes, qui n'ont cessé de se répercuter et de s'entremêler entre les champs de la linguistique et de l'histoire.

\section{Problèmes de terminologie}

Les termes de « sémitique », « couchitique », « chamitique », formés à partir des noms des descendants de Noé, sont chargés de représentations erronées de l'histoire de l'humanité, particulièrement de conceptions raciologiques obsolètes, formulées par les traditions exégétiques juives et chrétiennes, qui se sont perpétuées parmi les savants jusqu'au XIX ${ }^{\mathrm{e}}$ siècle (au moins), et plus longtemps encore chez les non-spécialistes ${ }^{5}$.

E. Renan, dans son Histoire générale et système comparé des langues sémitiques, ouvrage pionnier de la philologie comparée moderne, déplorait déjà l'emploi «défectueux » du terme sémitique, auquel il préférait la désignation «syro-arabe », mais il se pliait, non sans précautions, à la force de l'usage : «La dénomination de sémitique ne peut avoir d'inconvénients, du moment qu'on la prend comme une simple appellation conventionnelle et que l'on s'est expliqué sur ce qu'elle renferme de profondément inexact » (1855 : 2). Cependant, par une funeste prémonition, il reconnaissait que «ce nom, que l'usage nous oblige de conserver, sera longtemps la cause d'une foule de confusions » (1855:43).

Malgré les ravages de l'antisémitisme ou des théories de la supériorité chamitique en Afrique, la plupart des linguistes ont conservé la même attitude que leur illustre prédécesseur, pensant d'une part que toute désignation

5. Sur ces questions, voir B. LEwIS (1987: 49-69). Le tableau généalogique des descendants de Noé, donné au chapitre X de la Genèse correspondait sans doute à la représentation des peuples connus par les Hébreux à l'époque de la rédaction du texte biblique, sans construction d'une typologie raciale de l'humanité. Néanmoins l'épisode du chapitre précédent de la malédiction par Noé de Canaan, en raison de l'impudence de son père Cham, induisait déjà une idée d'inégalité entre peuples. Sur ce dernier point voir E. SANDERS (1969) et J.-P. Chrétien (1977). 
d'une entité aussi abstraite qu'une famille de langues est forcément conventionnelle (Cohen 1988 : 1) et cherchant, d'autre part, à neutraliser ces termes équivoques en les confinant à un sens strictement scientifique. Des dénominations alternatives ont été aussi proposées, telles «afro-asiatique », par J. Greenberg en 1952, ou « $\operatorname{lisramic}^{6}$ », la première ayant rencontré le plus de succès dans l'usage américain. Ce problème est cependant assez accessoire et peut nous empêcher de considérer la principale source de confusion qui consiste à prendre les classifications linguistiques pour des groupes identitaires pertinents: les Indo-Européens, les Bantu, les Sémites et autres Hamites sont des vues de l'esprit rationaliste moderne qui n'ont, jusqu'à récemment, jamais été des critères de reconnaissance ethnique.

\section{Autres résurgences bibliques}

Les théories de la linguistique génétique se sont inspirées des modèles généalogiques bibliques et ont perpétué quelques hypothèses exégétiques tronquées, pouvant aboutir à des confusions discriminantes. Mais en se dégageant peu ou prou de cette gangue, elles ont permis d'éclairer d'un jour nouveau l'histoire de l'humanité, en particulier là où les indices archéologiques étaient très rares.

Dans le cas éthiopien, les références bibliques, particulièrement à l'Ancien Testament, sont omniprésentes dans les représentations de l'histoire de cet État, et la conception philologique des « retrouvailles » de Sem et de Cham n'en est qu'un avatar moderne. Cette imprégnation vétéro-testamentaire est manifeste dans le nom même d'Éthiopie, dont l'étymologie renvoie au toponyme grec Aithiopia construit sur aithiops, signifiant littéralement «visage brûlé7 ». Par ce terme, les géographes grecs désignaient, au sens large, les peuples de peau noire, du sud de l'Égypte jusqu'à l'Inde, et, au sens plus restreint, les peuples habitant les régions du Haut Nil. C'est par ce nom que les traducteurs de la Bible en grec, les Septante, ont traduit le terme hébreu $K \hat{u} s h^{8}$, qui semble avoir désigné spécifiquement le royaume de Méroë, mais s'appliquait tout aussi vaguement à l'Afrique noire. Les traducteurs latins de la Vulgate ont repris ce nom sous la forme Athiopia. De la même façon, les traducteurs aksumites des Écritures ont, à partir du $\mathrm{IV}^{\mathrm{e}}$ siècle, transcrit le nom grec en langue guèze sous la forme Ityop'ya. Ces chrétiens se sont vraisemblablement reconnus dans cette terre sacrée, contournée par le Nil (Gihôn) (Genèse 2:13), et promise à une destinée glorieuse dans plusieurs passages de la Bible, notamment ce verset $32 \mathrm{du}$

6. Ce terme a été forgé par le linguiste C. T. Hodge (1972) à partir de racines lexicales propres à ce groupe : *lis : langue et *âmec : gens. Les * désignent des racines reconstruites selon des lois phonétiques mais non attestées.

7. De áithein : faire brûler et óps: visage.

8. k’š en égyptien ancien, ku:šo en assyrien, ku:su en babylonien, ku:ša en vieux-perse. 
psaume 68: «L'Éthiopie (Kush) tendra les mains vers Dieu. » De ce fait, le nom d'Éthiopie est devenu l'une des appellations savantes du royaume chrétien des hautes terres d'Afrique de l'Est. Cet usage s'est maintenu à travers une longue tradition de théologie politique, dans laquelle l'État, dirigé par une dynastie sacrée, héritière de l'alliance avec Dieu, est l'instrument du cours providentiel de l'histoire : «L'histoire de l'Éthiopie [est conçue dans la tradition salomonienne comme] au centre de l'histoire du salut de l'humanité. Les Éthiopiens, peuple élu, mènent la lutte du Bien contre le Mal en sachant que le Bien a triomphé et qu'il triomphera encore. Dieu envoie des épreuves pour s'assurer de la fidélité de son peuple, et, aussi, des hommes pour les surmonter. Face aux pires catastrophes, armés de telles certitudes, les Éthiopiens ont opéré des redressements "miraculeux" que les lettrés ont consignés dans leurs manuscrits, les agrégeant ensuite aux textes sacrés »(Gascon 1987 : 143).

Ce terrain idéologique fut particulièrement propice à toutes sortes de fantasmes grandiloquents, d'hypothèses hasardeuses et de revendications aberrantes, fondées sur des assemblages de matériaux savants et de traditions excessivement extrapolées. Après un aperçu des écueils sous-jacents de la linguistique des langues éthiopiennes, il est temps de faire état de l'extension contemporaine de la langue amharique, avant d'examiner sommairement l'histoire de ses relations à l'État.

\section{L'amharique dans tous ses états}

Selon les résultats du dernier recensement de la population éthiopienne, réalisé en $1994^{9}$, l'amharique est la langue «maternelle » de presque un tiers $(32,7 \%)$ des Éthiopiens, et la langue seconde de 10,4\% (84\% déclarant ne pas avoir de seconde langue). Parmi les locuteurs d'une seconde langue, une majorité importante $(61 \%)$ parle l'amharique.

En milieu urbain $(13,7 \%$ de la population), l'amharique est parlé par $80,5 \%$ des habitants $(56,5 \%$ en langue première, $24 \%$ en langue seconde). Si l'on soustrait du total la région Amhara et Addis Abeba (où l'amharique est parlé comme langue première respectivement par 93,2 \% et $72,6 \%$ ) on constate que les personnes dont l'amharique est la langue maternelle sont $7,9 \%$ de la population des autres régions. Près de la moitié $(47,7 \%)$ de

9. Ce recensement coïncidait avec la mise en place, depuis 1991, d'un nouveau régime en Éthiopie, organisé sur une base ethno-fédérale, c'est-à-dire un assemblage de régions, jouissant d'une certaine autonomie, constituées autour d'une ethnie ou «nationalité » majoritaire dont le critère distinctif est la pratique majoritaire d'une langue. Les résultats de cette enquête visaient donc à démontrer la fiabilité de ce nouveau découpage, au moment où était adoptée la constitution, fin 1994. De tels enjeux pouvaient laisser craindre des manipulations des chiffres pour simplifier des situations potentiellement litigieuses. Cependant, ayant comparé ces données statistiques à mes observations en diverses localités, je leur accorde un certain crédit. 
ces amharophones en situation d'allophonie vivent en milieu urbain. En outre, les autres habitants de ces régions sont $11,5 \%$ à pratiquer l'amharique en langue seconde, 27,8 \% d'entre eux vivant en ville. En tout, deux tiers $(65,5 \%)$ de la population urbaine de ces régions parlent l'amharique (35,4\% comme langue première et $30,1 \%$ comme langue seconde).

Ces chiffres montrent que l'amharique est la langue éthiopienne la plus largement diffusée ${ }^{10}$, bien qu'elle soit loin d'avoir une extension nationale homogène. Parlé sur un vaste ensemble territorial linguistiquement très cohérent sur les hautes terres centrales du pays, il rayonne hors de son «foyer» par les villes, au premier rang desquelles la capitale, située en plein pays de langue oromo. Malgré cette importance de l'amharique dans les activités économiques, politiques et sociales du milieu urbain, son usage n'a que superficiellement atteint les populations rurales.

Cette configuration linguistique reflète les grandes lignes de l'histoire du royaume chrétien de langue amharique et des autres sociétés devenues éthiopiennes en lui étant soumises.

L'Amhara était un territoire de la région centrale des hautes terres éthiopiennes, dont un souverain est devenu « roi des rois » à la fin du XIII ${ }^{\mathrm{e}}$ siècle, fondant une dynastie qui se réclamait de la descendance de Salomon et des rois d'Aksum. Les Salomonides d'Amhara mirent d'abord fin au pouvoir d'une autre dynastie chrétienne supposée d'ethnie agäw, avec le soutien de certains ordres monastiques et missionnaires. Ils contrôlèrent un vaste empire à l'assise montagnarde, exerçant leur hégémonie sur une grande partie des territoires du nord-ouest de la Corne de l'Afrique. Dans cet empire, le guèze conservait un usage liturgique et littéraire au sein du clergé, tandis que l'idiome des rois d'Amhara, l'amareñ̃a, et leur culture politique étaient devenus les attributs officiels d'un pouvoir temporel «dont les vicissitudes ont été variées : territoires acquis peu à peu, souvent reperdus et reconquis ; centre mobile représenté par une cour et une armée s'attachant temporairement à des capitales stables, dont les principales ont été fondées en dehors du milieu proprement amhara »(Cohen 1936 : 13-14). Cet exercice itinérant du pouvoir, la grande rotation des élites aristocratiques dans la hiérarchie militaire, l'allocation aux hommes de troupe de terrains sur les territoires conquis, l'affaiblissement des peuples dominés par la guerre et par la mise en esclavage, les parcours initiatiques à travers tout le pays des jeunes clercs de l'Église... sont autant de facteurs qui peuvent expliquer la relative uniformité de la langue amharique «non émiettée en parlers locaux et dont les variations provinciales sont peu importantes » (ibid.).

À partir du XIX ${ }^{\mathrm{e}}$ siècle, sortant de périodes successives de crise, de repliement et de désagrégation, l'empire chrétien amhara se recomposa et entrepris de conquérir les périphéries méridionales, occupées en grande partie

10. À titre de comparaison, l'oromo est la langue maternelle d'un autre tiers $(31,5 \%)$ des Éthiopiens, mais n'est la langue seconde que de $18 \%$ des locuteurs d'une langue seconde. 
par le peuple oromo. C'est l'empereur Ménélik (régnant de 1889 à 1913) qui paracheva cette conquête, donnant à l'Éthiopie ses frontières actuelles. L'ambition de Ménélik et de son successeur indirect, Hailé Sélassié (empereur de 1930 à 1974), était de faire de l'Éthiopie un véritable État-nation moderne, fondé sur une administration civile et militaire centralisée et omniprésente, prenant pour modèle unificateur la culture chrétienne du peuple amhara, et utilisant la langue amharique comme langue nationale dans le « rêve vain [pour une société agraire] de tenter d'imposer, à tous les niveaux de la société, une classe de clercs universalisée et une culture homogénéisée, avec des normes imposées du centre, renforcées par l'usage de l'écriture » (Gellner 1989: 33).

Cette politique s'est exercée au travers d'un maillage de villes-garnisons, relais du pouvoir central et pôles socio-économiques. En dépit de cette propagation très encadrée de la langue nationale, dont nous avons évalué les effets plus haut, les répulsions ont aussi été fortes contre cette imposition de la culture étatique et populaire amharique comme modèle de l'identité nationale éthiopienne : «Pour être un authentique Éthiopien, il faut parler l'amharique, écouter la musique amhara, accepter leur religion, le christianisme orthodoxe, et porter leur costume, la shama. Parfois, il faut aller jusqu'à changer de nom. En bref, et pour reprendre l'expression de Franz Fanon, pour être Éthiopien, il faut porter un masque amhara. Essayez d'affirmer votre identité nationale et vous serez automatiquement accusé de tribalisme; à moins que vous n'ayez la grâce d'être né amhara » (Walleligne Makonnen 1969).

Le centralisme éthiopien, dont l'amharique était l'un des vecteurs, s'est poursuivi après la destitution de l'empereur par la junte militaire, malgré quelques tentatives de prise en compte du caractère «multiculturel» de la société, en permettant l'usage public d'autres langues ${ }^{11}$ et promouvant les manifestations folkloriques tout en imposant de sévères entraves à la liberté d'expression.

Le parti dirigeant du régime éthiopien actuel, le Front révolutionnaire et démocratique du peuple éthiopien, est issu d'un mouvement sécessionniste d'un peuple du nord de l'Ethiopie, les Tegray ${ }^{12}$. En s'organisant en fédération, le nouvel État a laissé à chaque peuple le choix (à défaut des moyens) de sa propre politique linguistique, culturelle et d'éducation, prenant le risque, souvent dénoncé, de dissoudre une cohésion nationale si chèrement acquise. Malgré ces changements d'orientation, l'amharique a gardé une position prépondérante, tant par son statut de langue de travail

11. Sur la politique linguistique du régime révolutionnaire éthiopien à tendance marxiste, voir O. KAPELIUK (1980).

12. Ce peuple qui occupe aussi les hautes terres de l'Érythrée, a aussi été la composante principale du Front de libération d'Érythrée. La scission en deux fronts entre les Tegray d'Éthiopie et ceux d'Érythrée a été l'un des syndromes précurseurs de la récente guerre entre ces deux États. 
du gouvernement fédéral, que par ses fonctions de langue véhiculaire, surtout en milieu urbain, et de langue majeure de la presse et des médias. Plus significatif encore, le choix de la Fédération des nationalités du Sud, regroupant la mosaïque des peuples du sud-ouest, d'utiliser l'amharique comme seule langue commune administrative, permettant à ces minorités de s'exprimer d'une seule voix et d'être au moins entendues, sinon écoutées.

Cette présentation sommaire de l'expansion de la langue amharique est globalement satisfaisante. Nous pouvons revenir cependant sur quelques scories de notre raisonnement qu'il nous faut encore passer au crible. La schématisation de l'histoire éthiopienne présente en effet le risque de renforcer le fil conducteur dynastique, et de faire resurgir la conception, déjà partiellement décortiquée, d'une continuité et d'une supériorité, en somme d'une légitimité de la nature sémitique du pouvoir incarnée par la dynastie amhara salomonide. Notre critique portera brièvement sur deux illusions : celle de continuité dynastique et celle de prédestination nationale de l'amharique.

La dynastie salomonide a tenu le pouvoir impérial durant sept siècles, jusqu'au renversement de Hailé Sélassié en 1974, faisant preuve d'une longévité exceptionnelle en Afrique. Cette continuité est cependant une apparence produite par la mise en perspective historique. La lignée salomonide ne fut pas à l'abri des crises de succession, des révolutions de palais, de guerres intestines et de menaces extérieures, mais elle a pu perdurer malgré toutes ces ruptures, en raison même de l'idéologie providentialiste qui la soutenait, permettant à des princes éloignés de la filiation impériale directe, voire à des usurpateurs, de s'y rattacher en prétendant à une élection d'ordre divin révélée par différents signes (rêves, divination, interprétation onomastique). Si elle n'est pas fictive, la continuité de la dynastie salomonienne est donc factice pour une large part.

La fortune de l'amharique est intimement liée au projet impérial éthiopien, mais cette langue n'était pas destinée intrinsèquement à devenir la langue nationale éthiopienne, comme l'ont suggéré certains commentateurs un peu hâtifs, prenant sa nature mixte sémitique et couchitique pour preuve de sa dimension nécessairement «pan-éthiopienne $»^{13}$. De plus, l'histoire de la langue amharique n'est pas circonscrite à l'espace politique constitué par l'État chrétien, et il est possible de contourner la conception d'un monolithisme étatique de l'amharique en prenant en considération une société musulmane dont la langue offre un point de fuite intéressant.

\section{La tangente argobba}

Les Argobba forment aujourd'hui un ensemble social très fragmenté, dispersé en une succession de villages établis sur le rebord des hautes terres,

13. Cette thèse a été particulièrement appuyée par D. LEvine (1974 : 72) considérant que « le caractère composite de cette langue lui a donné une qualification prééminente pour devenir la langue nationale éthiopienne ». 
à leur limite encore cultivable. Malgré une extinction de cette société annoncée depuis longtemps par ses observateurs, elle existe toujours. En 1975, le géographe Völker Stitz (1975 : 188) en avait déjà fait le constat. Après avoir enquêté dans des villages argobba, il estimait à 6000 le nombre d'Argobba, dont il définissait ainsi l'identité : «En bref, Argobba désigne un sédentaire, dont l'activité principale est l'agriculture, le tissage ou le commerce, qui vit dans l'Yifat ou le Qallu [régions des contreforts orientaux du plateau du Choa], qui est musulman et qui parle soit l'argobba soit l'amharique. »

Les statistiques de 1994 amplifient considérablement ces estimations, car 62831 personnes se sont déclarées être d'ethnie argobba. La majorité $(60 \%)$ vit à l'est de la région Amhara, dans la zone Nord-Choa (43\%) et dans la zone Oromiya (17\%). Plus au Sud, ils sont près de 10000 à proximité de la vallée de l'Awash, dans la région afar, dans un district qui leur est propre, où se situe la direction du Mouvement démocratique des peuples argobba. Enfin, plus de 12000 vivent dans la région Oromo, sur les versants occidentaux du massif du C'erc'er. Les villages argobba au sud de la ville Harar, dont les descriptions de la fin du XIX ${ }^{\mathrm{e}}$ siècle et du début du XX $\mathrm{X}^{\mathrm{e}}$ suggéraient qu'ils se dépeuplaient et perdaient leur identité ${ }^{14}$, semblent avoir complètement disparu en tant que communautés distinctes.

Les locuteurs de la langue argobba sont beaucoup moins nombreux. Seulement 10860 (17,3\%) déclarent parler l'argobba comme langue maternelle, 4505 dans la région Amhara, soit $12 \%$ des Argobba de cette région, et presque autant dans la région afar, soit 44,5\% des Argobba de cette région, qui apparaissent beaucoup plus conservateurs, peut-être en raison de leur engagement politique plus prononcé. Ils ne sont que 671 en région oromo. Ces chiffres sont à peine rehaussés par les 3236 personnes qui déclarent utiliser l'argobba comme langue seconde.

Pourquoi faisons-nous cas de ce petit peuple, et de cette langue plus ténue encore face au colossal ensemble amhara ? La langue argobba est en fait si proche de l'amharique qu'elle peut en être considérée comme un dialecte, ouvrant la problématique qui nous semblait close précédemment. Les principaux travaux sur cette langue ont été l'œuvre des plus éminents spécialistes de l'amharique, mentionnés plus haut. Praetorius (1879: 14) incluait déjà à sa grammaire de l'amharique quelques remarques sur des vocabulaires d'argobba recueillis par les voyageurs Seetzen (1816) et Lefebvre (sd.) $)^{15}$. Dans ses Nouvelles études d'éthiopien méridional, travaillant sur des matériaux recueillis parmi les derniers locuteurs d'argobba méridional par Mme de Monfreid, Marcel Cohen (1939: 427) restait prudent sur le degré de proximité dialectale entre les deux langues, constatant que

14. Voir M. Cohen $(1931,1939)$ pour une recension de ces sources. W. LeSLAU (1997 : 1) a aussi fait ce constat à ses dépens en 1948, revenant bredouille de son enquête sur l'argobba méridional.

15. Dans son examen de ces vocabulaires, W. Leslau (1949) a montré qu'ils décrivent plus probablement la langue selti-walani du groupe guragé, que la langue argobba, au sens où on l'entend aujourd'hui. 
«l'argobba est par divers traits grammaticaux tout proche de l'amharique, mais s'en écarte nettement par d'autres » et attendait que des données plus consistantes soient fournies pour statuer sur cette question. Après avoir lui-même travaillé avec des informateurs argobba à Addis Abeba ou dans le Choa, W. Leslau a publié plusieurs articles $(1949,1957,1959,1966)$ sur cette langue qu'il a complétés par les données d'une récente « enquête sur les langues peu connues d'Ethiopie» (Survey... 1994), entreprise par des linguistes de l'Université d'Addis Abeba pour mettre au point en 1997 la première grammaire complète de l'argobba, suivie d'un dictionnaire de 1300 entrées. Achevant son analyse par un examen comparé des systèmes grammaticaux de l'amharique et de l'argobba, il concluait : «L'intercompréhension combinée à de nombreux traits communs entre l'amharique et l'argobba m'amènent à conclure que l'argobba est un dialecte amharique. Ce qui est particulièrement intéressant est que l'argobba a conservé certains traits archaïques que l'amharique contemporain n'a pas » (Leslau 1997: 131).

Il corroborait cette conclusion par une preuve lexicographique montrant que $87 \%$ du lexique argobba qu'il a collationné est commun avec l'amharique. Pour V. Stitz (1975 : 191) « la séparation des deux dialectes eut lieu il y a environ mille ans, selon les résultats de la méthode glotto-chronologique », sans indiquer malheureusement sur quelles sources il se fondait.

Ces arguments linguistiques nous suggèrent que l'argobba dérive d'un état ancien de l'amharique, avant que le parler de l'Amhara ne connaisse l'essor que nous avons décrit. Les conséquences de cette hypothèse sont importantes pour la linguistique et l'histoire éthiopiennes mais n'ont pas été suffisamment tirées.

D'abord, les divergences que présente l'argobba par rapport à l'amharique (Leslau 1997 : 116-131) permettent de mesurer à quel point l'amharique contemporain est effectivement uniforme. Les évolutions morphologiques qu'il a subies ont en effet été répercutées sur tous ses parlers régionaux, telles :

- l'infixation de -ačč- au possessif pluriel ;

- l'effacement complet des consonnes laryngales étymologiques dans les racines verbales, alors que l'argobba conserve un $h$ substitut;

- l'indistinction vocalique des deux types (dits A et B) de racines trilitères, alors que dans l'argobba, et probablement l'amharique ancien, le type B se distingue par une syllabe initiale vocalisée par un $e ́$;

- le développement des formes composées de l'inaccompli et du gérondif avec l'auxiliaire allä.

L'observation de l'argobba donne aussi les moyens de mieux identifier et de comprendre les quelques traits dialectaux qui apparaissent néanmoins en amharique et de conjecturer sur leur possible renforcement ou leur effacement en fonction des contextes sociaux où ils apparaissent ${ }^{16}$.

16. Ma recherche doctorale, en cours, porte en partie sur l'amharique des musulmans de la région du Wällo, qui tend par la religion, le commerce et l'émigration à intégrer de nombreux mots arabes et quelques traits articulatoires de cette langue. 
Sur un plan historique, la séparation des parlers argobba et amharique est une clé très utile à la compréhension du haut Moyen Âge éthiopien. Commençant par le lent déclin du royaume d'Aksum, amorcé par l'émergence de l'empire arabo-musulman au $\mathrm{VII}^{\mathrm{e}}$ siècle, cette période est conçue classiquement comme une sorte d'hibernation de la civilisation aksumite, repliée sur elle-même face aux débordements de peuples «couchitiques » Béja et Agäw, et à l'encerclement économique et idéologique exercé par des États musulmans. Elle serait sortie de cette adversité multiple à la fin du XIII ${ }^{\mathrm{e}}$ siècle, transformée, métissée et magnifiée par les souverains salomonides d'Amhara.

Cependant, au moment où s'est formée cette dynastie chrétienne, les locuteurs d'une langue proto-amharique ne formaient pas un ensemble ethnique compact, issu d'une colonie aksumite méridionale, isolée dans des montagnes imprenables, et tout entière tournée vers la reconquête du pouvoir, contre des usurpateurs d'une race inférieure (une dynastie dite Zagwé, que l'on suppose d'ethnie agäw avait en effet récupéré l'exclusivité du sacre chrétien). Une partie de ce peuple «proto-amhara» était établie sur les versants des plateaux. Cet escarpement est, par endroits, assez abrupt et peut sembler être un rempart mais en d'autres il est beaucoup moins prononcé, formant des paliers étendus, qui créent un espace de circulation et d'échanges très actifs entre les hautes terres agricoles et les basses terres désertiques débouchant sur la mer Rouge. Les nombreuses places de marché qui jalonnaient ce territoire interstitiel ont permis à ce peuple de prendre le contrôle d'une partie des routes caravanières. Loin des côtes, restant implantée sur des reliefs protecteurs et salubres, cette société s'est rattachée à un vaste réseau dont l'islam fournissait en quelque sorte les codes d'accès culturels et idéologiques. Attirés par les possibilités d'un négoce lucratif parmi des populations fraîchement converties, des hommes venant d'Arabie, plus ou moins pétris de science islamique, s'attribuant une ascendance prestigieuse dans la lignée du prophète, ont gagné une forte respectabilité, occupant des positions de pouvoir jusqu'à devenir des souverains fondateurs de dynasties conquérantes.

Nous venons d'exposer brièvement les fondements des États musulmans du rebord des hauts plateaux, dont les Argobba anciens formaient hypothétiquement la population majoritaire ${ }^{17}$. Ils vivaient probablement dans le sultanat du Choa — dont la dynastie Makhzûmî aurait commencé à régner au

Les contacts avec les langues d'autres communautés musulmanes de la région (oromo, afar) peuvent aussi être détectés.

17. Quelques données sur les traditions orales argobba (AKLILU Asfaw 2000 ; TEsFAYE Hailu 2000) et sur des sites archéologiques argobba (KebBede Geleta 2000) ont été récemment publiées dans le volume 16 des Annales d'Éthiopie. Sur l'identité argobba contemporaine notons l'existance d'une thèse (ABEBE KifLEYesus 1992) et d'un article traitant spécifiquement des pratiques de l'islam mystique (AbeBe Kifleyesus 1995). 
début du $\mathrm{x}^{\mathrm{e}}$ siècle —, et plus certainement dans celui d'Yifat, dont la dynastie Walashma' a renversé la précédente et conquis ses territoires et positions commerciales à la fin du XIII ${ }^{\mathrm{e}}$ siècle $^{18}$. Au même moment, le premier souverain salomonide d'Amhara imposait son autorité aux provinces chrétiennes. Cette coïncidence peut être l'indice d'une scission entre les Amhara christianisés et Argobba islamisés, qui entretenaient auparavant des relations complémentaires, avant de se lancer dans des politiques hégémoniques concurrentes. Les relations entre ces deux dynasties se sont vite dégradées, ayant pris de part et d'autre la dimension d'une guerre sainte, qui a atteint son paroxysme au $\mathrm{XVI}^{\mathrm{e}}$ siècle.

Nous n'entrerons pas plus avant dans les détails de cette histoire tumultueuse. Nous avons seulement cherché à souligner que l'existence d'une société islamisée de langue proto-amharique, les Argobba, nous donne un éclairage contraire à la vision sacralisée et unifiante de la langue nationale éthiopienne. Ces quelques suggestions visent à corroborer des recherches actuellement entreprises en archéologie et en histoire médiévale sur les dynamiques qui ont préparé la constitution de l'empire salomonide et sur ses relations au monde arabo-musulman.

Cette pluralité sous-jacente de l'amharique révélée par la prise en considération de l'argobba ne doit pas être seulement envisagée comme un état ancien des choses, une mixité originelle de laquelle auraient émergé au cours de l'histoire des blocs homogènes. Si l'amharique a atteint un certain degré d'uniformité et de véhicularité, lié comme nous l'avons vu à son usage par les cercles du pouvoir, des processus inverses de diversification ont aussi existé, et sont toujours à l'œuvre, mais n'ont été que peu relevés. D'où une nécessité de collaboration suivie entre linguistes, historiens et anthropologues sur ce terrain fertile.

Centre d'études africaines, EHESS.

18. Pour une histoire générale de l'islam en Éthiopie à l'époque médiévale, voir J. Cuoq (1981). 


\section{BIBLIOGRAPHIE}

\section{Abebe Kifleyesus}

1992 The Dynamics of Ethnicity in a Plural Polity: Transformation of Argobba Social Identity (Ethiopia), Ph.D., Northwestern University.

1995 «Sufism and the Rural and Urban Reality of Argobba Mysticism », Islam et Sociétés au Sud du Sahara, 9: 27-46.

AkLilu Asfaw

2000 «A Short History of the Argobba », Annales d'Éthiopie, $16: 173-183$.

Amsalu Aklilu

1991 "The Influence of Arabic on Wollo Amharic», in A. S. KAYE, ed., Semitic Studies in Honor of Wolf Leslau on the Occasion of his 85th Birthday, Wiesbaden, Harrassowitz Verlag: 72-81.

Amsalu Aklilu \& Habte Mariam Marcos

1973 « The Dialect of Wällo », in Habte Mariam Marcos, ed., « Regional Variations in Amharic », Journal of Ethiopian Studies, 11 (2) : 124-129.

ApPleyARD, D.

1978 «Linguistic Evidence of non-Semitic Influence in the History of Ethiopian Semitic: Lexical Borrowing in Ge'ez and Other Ethiopic Semitic languages », Abbay, $9:$ 49-56.

1979 «A Statistical Survey of the Amharic Lexicon», Journal of Semitic Studies, 24 : 71-97.

BENDER, M. L.

1971 «The Languages of Ethiopia: A New Lexicostatistic Classification and Some Problems of Diffusion », Anthropological Linguistics, 13 (5) : 165-288.

1972 «Loanwords in Amharic Daily Newspaper», Anthropological Linguistics, $14(8)$ : 317-322.

BENDER, M. L., ed.

1976 The Non-Semitic Languages of Ethiopia, East Lansing, African Studies Center, Michigan State University.

Bender, M. L. \& Hailu Fulass

1978 Amharic Verb Morphology, East Lansing : African Studies Center, Michigan State University.

Bender, M. L., Bowen, R. L., Cooper, C. A. \& Ferguson, C. A., eds

1976 Language in Ethiopia, London, Oxford University Press.

Chrétien, J.-P.

1977 «Les deux visages de Cham. Points de vue français sur les races africaines d'après l'exemple de l'Afrique orientale ", in P. Guiral \& E. Termime, eds, L'idée de race dans la pensée politique française, Paris, Éditions du CNRS : 171-199. 
COHEN, D.

1988 «Introduction », in D. CoHEN, ed., Les langues chamito-sémitiques, $3^{\mathrm{e}}$ partie de J. Perrot, ed., Les langues dans le monde ancien et moderne, Paris, Éditions du CNRS : $1-8$.

COHEN, M.

1931 Études d'éthiopien méridional, Paris, Geuthner.

1936 Traité de langue amharique (Abyssinie), Paris, Institut d'ethnologie.

1939 Nouvelles études d'éthiopien méridional, Paris, Honoré Champion.

1947 Essai comparatif sur le vocabulaire et la phonétique du chamito-sémitique, Paris, Honoré Champion.

COOPER, R. L.

1976a «Government Language Policy », in M. L. BENDER et al., Language in Ethiopia, op. cit. : 187-190.

$1976 b$ «The Spread of Amharic», in M. L. BENDER et al., op. cit. : 289-301.

CuOQ, J.

1981 L'Islam en Éthiopie: des origines au XVI ${ }^{e}$ siècle, Paris, Nouvelles éditions latines.

Drewes, A. S.

1970 «Bilingualism in Ethiopia», in Proceedings of the Third International Conference of Ethiopian Studies, Addis Abeba, Institute of Ethiopian Studies, vol. 2 : $57-60$.

Federal Democratic Republic of Ethiopia

1998 The 1994 Population and Housing Census of Ethiopia. Results at Country Level, vol. 1 : Statistical Report, Addis Abeba, Central Statistical Authority, Office of Population and Housing Census Commission.

Fellman, J.

1976 «The Resolution of Diglossia in an African Setting: The Ethiopian Case », La Monda Linguo-Problemo, 5 : 178-183.

1977 «A Correlation Between Language and Culture : The Amharic Case », Orbis, 27 (2) : 241-243.

1984 «Language, Geography, and History: The Case of Ethiopian Semitic », Anthropos, 77 (1-3) : 256-257.

1992 «Language and National Identity: Amharic in Ethiopia», Research in African Literatures, 23 (1): 173-174.

Ferguson, C. A.

1971 «The Role of Arabic in Ethiopia : A Sociolinguistic Perspective », in A. DiL, ed., Language Structure and Language Use, Stanford, Stanford University Press : 293-312.

GAsCON, A.

1987 «État ancien, État contemporain : l'Éthiopie », in E. TERrAy (dir.), L'État contemporain en Afrique, Paris, L'Harmattan : 141-156. 
Gellner, E.

1989 Nations et nationalisme, Paris, Payot.

Getachew Haile \& Seifu Metaferia

1973 « The Dialect of Gojjam », in Habte Mariam Marcos, ed., op. cit. : 113-120.

Habte Mariam Marcos, ed.

1973 «Regional Variations in Amharic », Journal of Ethiopian Studies, 11 (2) : 113-129.

Hailu Fulass \& Fisseha Sisay

1973 « The Dialect of Mänz », in Habte Mariam Marcos, ed., op. cit. : 120-124.

Hodge, C. T.

1972 «Lisramic », Language Sciences, 20 : 13-16.

KAPELIUK, O.

1980 «Language Policy in Ethiopia since the Revolution of 1974 », Asian and African Studies, 14 (3) : 269-278.

\section{KebBede Geleta}

2000 «A Survey on Argobba Sites in Northern Shoa», Annales d'Éthiopie, 16 : 185-194.

LEFEBVRE, T.

s.d. Voyage en Abyssinie exécuté pendant les années 1839-1843, Paris, Arthus Bertrand [vocabulaire «argobba », vol. $3: 405-409$ ].

LESLAU, W.

1949 «Examen du supposé argobba de Seetzen et de Lefebvre», Word, 5 (1) : 46-54.

1957 «Arabic Loanwords in Argobba », Journal of the American Oriental Society, 77 (1) : 36-39.

1959 «A Preliminary Description of Argobba », Annales d'Éthiopie, 3 : 252-272.

1965 An Annotated Bibliography of the Semitic Languages of Ethiopia, LondonThe Hague-Paris, Mouton.

1966 «An Analysis of the Argobba Vocabulary », Journal of African Languages, 5 (2) : 102-112.

1990 Arabic Loanwords in Ethiopian Semitic, Wiesbaden, Harrassowitz Verlag.

1995 Reference Grammar of Amharic, Wiesbaden, Harrassowitz Verlag.

1997 Ethiopic Documents : Argobba. Grammar and Dictionary, Wiesbaden, Harrassowitz Verlag.

LEVINE, D. N.

1974 Greater Ethiopia. The Evolution of a Multiethnic Society, Chicago-London, University of Chicago Press.

LEwIS, B.

1987 Sémites et antisémites, Paris, Fayard. 
PRAETORIUS, F.

1879 Die amharische Sprache, Halle.

1889 «Hamitische Bestandheile im Aethiopischen», Zeitschrift der morgenländlischen Gesellschaft, 43 : 317-326.

RENAN, E.

1855 Histoire générale et système comparé des langues sémitiques, Paris, Michel Lévy frères.

SANDERS, E. R.

1969 «The Hamitic Hypothesis : its Origin and Functions in Time Perspective », Journal of African History, 10 (4) : 521-532.

SEETZEN, U. J.

1816 Proben deutscher Volksmundarten. Dr Seetzens linguistischer Nachlass, und Andere Sprach-Forschungen und Sammlungen besonders über Ostindien, herausgegeben von Dr. Johann Severin Vater, Leipzig [vocabulaire « argobba » : 301-303].

STITZ, V.

1975 «The Western Argobba of Yifat, Central Ethiopia», in H. MArcus, ed., Proceedings of the First United States Conference of Ethiopian Studies, East Lansing, African Studies Center, Michigan State University : 185-192.

SuRVEY OF LitTLE-KNOWN LANGUAGES OF ETHIOPIA

1994 Linguistic Report, 22 [Argobba], Addis Abeba, Addis Abeba University, Institute of Ethiopian Studies.

Tesfaye Hailu

2000 «History and Culture of the Argobba: Recent Investigations », Annales d'Éthiopie, 16 : 195-206.

TUBIANA, J.

1959 «Note sur la distribution géographique des dialectes Agaw », Les Cahiers de l'Afrique et de l'Asie, 5 : 297-306.

1984 «Aperçus sur l'enrichissement du vocabulaire amharique », in I. FodoR \& C. HAGĖGe, eds, Language Reform : History and Future, Hamburg, Bukse, III : 331-365.

1985 «Brave New Words : Linguistic Innovation in the Social and Economic Vocabulary of Amharic », Journal of Semitic Studies, 30 (1) : 85-93.

1986 «Modernisation et emprunts lexicaux en amharique», in G. GoldENBERG, ed., Ethiopian Studies. Proceedings of the 6th International Conference, Rotterdam, Balkema: 501-508.

ULLENDORF, E.

1973 The Ethiopians. An Introduction to Country and People, 3rd ed., Oxford, Franz Steiner.

WALLELIGNE MAKONNEN

1969 «On the Question of Nationalities », Struggle, 5 (2) : 4-7. 


\section{RÉSUMÉ}

En Éthiopie, l'amharique peut être qualifié de «langue des langues » tant son expansion fut liée au destin de l'ancien empire chrétien et tant son usage reste prépondérant dans l'État fédéral contemporain. Cette langue présente une forte apparence d'uniformité qui a été peu mise en question. Un déni d'hétérogénéité dialectale semble en effet soutenir ce pilier de l'identité nationale éthiopienne. Les idéologies providentialistes et unitaristes qui ont imprégné I'histoire politique ont aussi déteint sur les travaux de la linguistique comparée chamito-sémitique. Pour échapper à ce cercle de représentations, l'examen de la langue argobba, parlée aujourd'hui par de rares communautés musulmanes, offre des ouvertures intéressantes. La reconnaissance de cette langue comme dialecte d'un état ancien de l'amharique permet en effet de concevoir une pluralité ancienne des réseaux d'identités et de pouvoirs auxquels participent les locuteurs d'amharique et donne un éclairage alternatif à la vision sacralisée et unifiante de la langue nationale éthiopienne.

\section{ABSTRACT}

Discovering the Amharic: Constrasting Ethiopian Languages and Histories. Amharic can be said to be the "language of languages" in Ethiopia, since its expansion was linked to the ancient Christian empire's destiny and it is still the major language used in the contemporary federal state. This language appears to be quite uniform, a uniformity that has more or less gone unquestioned. This denial of diversity seems to be a pillar supporting a national Ethiopian identity. Furthermore, the providentialistic, unitarian ideologies infused in political history have diffused into studies in comparative HamitoSemitic linguistics. An interesting way to escape from this constraint is to examine Argobba, a language spoken nowadays by the few Muslim communities in the country. Recognizing this language to be a dialect corresponding to a former state of Amharic enables us to imagine, for the past, a plurality of networks of identities and power, wherein Amharic-speakers took part. It presents us with an alternative view to the sanctified, unifying vision of the Ethiopian national language.

Mots-clés/Keywords : Éthiopie, Amhara, Argobba (peuple et langue), amharique, chamito-sémitique, dynastie salomonide chrétienne, islamisation, linguistique comparée/ Ethiopia, Amhara, Amharic, Argobba (the people and language), Hamitic/Semitic linguistics, the Christian Solomonid dynasty, islamization. 